\title{
FINITE PROJECTIVE DIMENSION UNDER CHANGE OF RINGS
}

\author{
PETER KOHN
}

Abstract. Suppose the local, central $R$-algebra $S$ is finitely projectively resolvable as an $R$-module, and that $A \neq 0$ is a finitely projectively resolvable $S$-module. It is proved that

$$
d_{R}(A)=d_{R}(S)+d_{S}(A),
$$

where $d$ is projective dimension. A well-known theorem of Auslander and Buchsbaum (Ann. of Math. 68 (1958) p. 634; MR 20 \#6414) is the Noetherian ring-theoretic case.

1. Introduction. Suppose the associative ring $S$ to be a unital $R$ algebra and its projective dimension as an $R$-module to be $n$. This way all $S$-modules become $R$-modules, and one wonders about the case of $S$ modules whose projective dimension over $R$ is precisely $n$ greater than their dimension over S. A well-known theorem of Auslander and Buchsbaum provides sufficient conditions for this case when $R$ and $S$ are commutative, local and Noetherian. We present a more general version with a more purely homological proof.

Statement and proofs in this paper are all at the elementary level of homological algebra, using Ext. All rings will have unit and be associative. For illustration let $T$ be a ring and $A$ a $T$-module. Modules will be unitary left modules $\left.{ }_{r} A\right)$ except as indicated otherwise: $A_{T}$. We will use the functor Ext, especially as a derived functor of Hom in its first variable. We will use projective dimension, noted $d_{T}(A)$, an invariant of the $T$-module $A$, which is the shortest length of a projective resolution of $A$. That is, $d_{T}(A)=0$ means $A$ is a projective module; $d_{T}(A)=m$, $\infty>m>0$, means $A$ is the image of an epimorphism from a projective module for which the kernel is $K$ and $d_{T}(K)=m-1$; and $d_{T}(A)=\infty$ means neither of the preceding. This is the only dimension we will use. If $m=d_{T}(A)<\infty$, then in any projective resolution of $A$, the $m$-th kernel must be projective. Also $\operatorname{Ext}^{j}(A,-)$ is identically zero if and only if $j>m$. If $A$ possesses a resolution of finite length by finitely generated projective modules, then any attempt to resolve $A$ by finitely generated projective modules is fated to success at the $d_{T}(A)$-th step,

Received by the editor on December 21, 1974.

This paper extends material in the author's Ph.D. thesis, written under Irving Kaplansky, and whom the author thanks for good stuff. The author received an Indiana University research grant during the paper's composition.

Key words and phrases: Projective dimension; change of rings; local rings; local algebras. 
and we say $A$ is FPR. There is also a concept of finitely freely resolvable, or FFR, modules. Some of these facts may be learned in [2, pp. 209-110], [4, pp. 167-169], [5, pp. 161-164] or [6, pp. 209-216]. The reader should also know that finitely generated projective modules over local rings [2, p. 147] are free. (Adapt [4, p. 182] or [2, pp. 154-156].)

When we say the ring $S$ is an $R$-algebra we mean there is a distinguished unital ring homomorphism from $R$ to the center of $S$. By $\operatorname{Ext}(A, B)$ we will always mean $\operatorname{Ext}_{R}\left({ }_{R} A,{ }_{R} B\right)$, even when $A$ was first an $S$-module. If $R$ and $S$ are local, commutative, Noetherian rings and the $R$-algebra $S$ is finite-dimensional and finitely generated as an $R$-module, then for any non-zero, finite-dimensional, finitely generated S-module, A,

$$
\mathrm{d}_{R}(A)=d_{R}(\mathrm{~S})+d_{S}(A) .
$$

This is well-known $[1$, p. 634] or [3, p. 129]. Our replacement follows.

THEOREM. Let the local ring and R-algebra $S$ be FPR as an R-module. If $A \neq 0$ is an FPR S-module, then it is FPR over $R$ and

$$
d_{R}(A)=d_{R}(S)+d_{S}(A) .
$$

2. Arguments. Part of this theorem is a commonplace and could well have introduced this paper. We state it as a double lemma. See [2, p. 360] and [3, p. 147].

LeMma 1. Let $S$ be an R-algebra (FPR as an R-module), and $A$ an (FPR) S-module. Then $d_{R}(A) \leqq d_{R}(S)+d_{S}(A)$. (A is an FPR R-module.)

Proof. Assume $d_{S}(A)<\infty$. Our induction on this quantity is the same as in the proof of the theorem but for the need there to work over $d_{S}(A)=1$. For here, let $0 \rightarrow K \rightarrow F \rightarrow A \rightarrow 0$ be a short exact sequence of $S$-modules with $F$ (finite) free over $S$. A basic theorem relating the dimensions (being FPR) of $K, F$ and $A$ is well-known [4, p 169], [5, p. 163], [6, p. 215]. Over $S$, we conclude $d_{S}(K)=d_{S}(A)-1(K$ is FPR). Applying induction, $d_{R}(K) \leqq d_{R}(S)+d_{S}(K)$ ( $K$ is FPR over $R$ ) and hence the desired conclusion with the basic dimension theorem.

Our task is now to prove actual equality of dimension in the theorem's case. For this we need Ext. Even in the situation of lemma 1 note that for any ${ }_{R} B, \operatorname{Ext}(S, B)$ is a left $S$-module since right multiplication of $S$ on $S$ is an $R$-homomorphism of ${ }_{R} S$ and by Ext acting contravariantly this action of $S$ on the right becomes an action best interpreted as on the left of $\operatorname{Ext}(S, B)$. See [2, pp. 22]. In the same way it is an $R$-module, which is the same way Ext is an $R$-module when $R$ is commutative. 
Lemma 2. Suppose $R$ commutative and its module $B \neq 0$ to be FPR, with $d_{R}(B)=n$. Denote by $C$ the $n$-th kernel in a finite projective resolution of $B$. Then $\operatorname{Ext}^{n}(B, C)$ is non-zero and finitely generated as an $R$ module. (See [2, pp. 122-123, exs. 2 and 9]).

Proof. Let $0 \rightarrow K \rightarrow P \rightarrow B \rightarrow 0$ be short exact with $P$ finitely generated projective. The proof is by induction on $n$. For $n=0$, further require $P$ to be free. Then $C=B$ and $\operatorname{Ext}^{n}(B, C)$ is a summand of $\operatorname{Hom}(P, P)$. Here one is not free to choose any projective as $C$ (Consider the case of $B$ a proper summand of $R$.), though $C=R$ would do. For $n=1, K=C, \operatorname{Ext}^{0}(P, K) \rightarrow \operatorname{Ext}^{0}(K, K) \rightarrow \operatorname{Ext}^{1}(B, K) \rightarrow \operatorname{Ext}^{1}(P, K)=0$ is an exact sequence of $R$-modules. By the case $n=0$, $\operatorname{Ext}^{0}(K, K)$ is finitely generated and the image of $1_{K}$ is non-zero else $d_{R}(B)=0$. For $n>1$, the similar sequence is $0 \rightarrow \operatorname{Ext}^{n-1}(K, C) \rightarrow \operatorname{Ext}^{n}(B, C) \rightarrow 0$, where $C$ is also the $n$-1-th kernel of $K$.

In case $R$ is Noetherian and $B$ is finitely generated, the result was well-known.

Corollary. Suppose the R-algebra $S$ is an FPR R-module, $d_{R}(S)=n$. Then, for some R-module $C, \operatorname{Ext}^{n}(\mathrm{~S}, C)$ is non-zero and finitely generated over $S$.

Proof. If $\operatorname{Ext}^{n}(S, C)$ is finite over $R$, the same generators do over $S$.

Proof of Theorem. Let $n=d_{R}(\mathrm{~S})$. We proceed by induction on $d_{S}(A)$, to show $d_{R}(A)=n+d_{S}(A)$. Since $S$-projectives are free, assume $d_{S}(A)=1$. (To show $d_{R}(A) \nless n+1$.) Taking $v$ as a number of a minimal generating set for ${ }_{s} A,{ }_{s}{ }^{A} \cong$ Coker $\left(f:{ }_{s} S^{u} \rightarrow{ }_{S} S^{v}\right), f\left(S^{u}\right) \subseteq M S^{v}$, where $u$ is finite and $M$ is the maximal ideal of $S$. That is, $f$ is a matrix whose entries are in $M$ and to be regarded as acting on the right. Also, $f$ is an $R$-homomorphism. With $C$ as in the Corollary to Lemma 2, $\operatorname{Ext}^{n+1}(A, C)$ as a group is the cokernel of

$$
\operatorname{Ext}^{n}(f, C): \operatorname{Ext}^{n}\left(S^{v}, C\right) \rightarrow \operatorname{Ext}^{n}\left(S^{u}, C\right) .
$$

Since Ext respects direct sums [2, p. 19] this is just the transpose of $f$,

$$
f^{t}:\left[\operatorname{Ext}^{n}(S, C)\right]^{v} \rightarrow\left[\operatorname{Ext}^{n}(S, C)\right]^{u} .
$$

the entries of $f^{t}$ acting now on the left. Even if $\operatorname{Ext}^{n+1}(A, C)$ is not an $S$-module it has as a group homomorphic image the $S$-module $\left[\operatorname{Ext}^{n}(S, C)\right]^{u} / M\left[\operatorname{Ext}^{n}(S, C)\right]^{u}$, which is non-zero by Nakayama's lemma. For $d_{S}(A)>1$ the induction step as in lemma 1 allows the desired conclusion. 
3. Discussion. Our theorem is of the following type. One specifies a type of finite-dimensional $R$-algebras, $S$. If $S$ is "close enough" to $R$ one can specify a class of finite dimensional non-zero $S$-modules for which our conclusion holds. The reader here must know the other change of rings theorem [3, p. 172], that the sum formula holds for any non-zero finite-dimensional $S$-module when $S=R /(x), x$ a central non-unit, nonzero divisor of $R$. Its corollaries [2, p. 150], [3, p. 127] provide the first examples of finite dimensional modules. This other theorem is notable for lack of finiteness conditions on $A$ and locality of $S$. Of the several counterexamples the reader should have at hand to illustrate the two theorems' difference and the extent to which they are best possible we provide three based on the beautiful non-regular ring, $k[x, y] /\left(x^{2}, x y\right)$, which all should recognize as of dimension 2 as a $k[x, y]$-module.

The first has to do with the finiteness of $A$. Let $R$ be polynomials in commuting variables $x$ and $y$ over a field $k$, localized to preserve $(x, y): R=k[x, y]_{(x, y)}$. Let $S=R /\left(x^{2}, x y\right)$. Adjoining $1 / y$ to $S$ gives $S[1 / y]=A=(R /(x))[1 / y]$. To calculate the dimension of $A$, take a resolution of $A$ sending free generators to $1 / y^{n}$, yielding a free relation module. For the $R$-dimension, do this over $R /(x)$ and then use the "other" change of rings theorem. Then we see $d_{R}(A)$ lacks equalling $d_{R}(S)+d_{S}(A)$ by one.

The second example is in regard to the locality of $S$. The global dimension, say of an FPR module $A$ over a commutative ring $T$, is the supremum of the dimensions of $A_{M}$ over $T_{M}$ for maximal ideals $M$. So, in a special case one could globalize but our example will show one is generally stuck with $S$ local, which even implies that the image of $R$ is local, provided $S$ is central over $R$. Let $R=k[x, y], S=R /\left(x^{2}, x y\right)$, $A=S /(y-1)=R /(x, y-1)$. $R$-sequence facts show that $d_{R}(A)=2$, $d_{R}(S)+d_{S}(A)=3$. Localizing to preserve $(x, y)$ and $(x, y-1)$ will yield a semi-local example.

The third example has to do with the common condition that $d_{R}(S)<\infty$. Let $R=k[x, y] /\left(x^{2}, x y\right), \quad S=R /(x) \cong k[y]$ and $A=S /(y-1)$ again. To see that $d_{R}(S)=\infty$, either adapt [4, p. 168] or localize at $(x, y)$ and apply [1, pp. 605-1], [3, p. 140].

This last example is deficient, however since $S$ is not local. By the same method that proved our theorem we can prove the following proposition, of particular interest when $d_{R}(S)=\infty$.

Proposition. Suppose $R$ is a commutative Noetherian ring and $S$ is a commutative local R-algebra, finitely generated as an R-module. If $d_{S}(A)=1$, where $A$ is a finitely generated S-module, then $d_{R}(A) \geqq d_{R}(S)+1$. 
Note that if the hypotheses of this proposition or of the theorem are altered so that $S$ is the $R$-algebra $R / I, I$ an ideal, but need not be local, one obtains $d_{R}(A) \geqq d_{S}(A)$.

\section{REFERENCES}

1. M. Auslander and D. A. Buchsbaum, Codimension and multiplicity, Ann. of Math. 68 (1958), 625-657.

2. H. Cartan and S. Eilenberg, Homological algebra (Princeton Univ. Press, 1956).

3. I. Kaplansky, Fields and rings (University of Chicago Press, Chicago, 1970).

4. Commutative rings (Allyn and Bacon, Boston, 1970).

5. R. E. MacRae, On an application of the Fitting invariants, J. Algebra 2 (1965).

6. J. Ohm, Homological dimension and Euler maps in Conference on Commutative algebra. Lawrence, Kansas, 1972 (Springer-Verlag, Berlin, 1973) pp. 209-221.

De Paul University, Chicago, Illinois 60614 
